\title{
论 文 系统可观测性理论与深空探测自主导航方法专题
}

\section{基于序列图像的深空小天体运动状态估计}

\author{
北京空间飞行器总体设计部, 北京 100094 \\ *联系人, E-mail: gedm1982@163.com
}

邓润然，葛东明 ${ }^{*}$ ，史纪金金，邹元杰，朱卫红，李佳宁

收稿日期: 2021-01-05; 接受日期: 2021-03-02; 网络出版日期: 2021-08-16

国家重点研发计划(编号: 2019YFA0706500)和国家自然科学基金(编号: U20B2055, 61525301, 61690215, 61640304, 61573060, 61203093)资助 项目

\begin{abstract}
摘要近年来, 随着空间技术的进步, 大部分国家逐渐开始关注深空小天体探测, 这属于典型空间非合作目标探 测任务. 与合作目标相比, 空间非合作目标具有典型的特征, 如无先验知识、无合作标识和无通信等. 但是在附着 之前, 必须获取空间非合作目标的必要信息. 如何通过非接触的方式获取目标的姿态、角速度和惯性参数是深空 小天体探测的重要技术之一。研究工作提出了一种估计未知非合作目标姿态、运动与惯量特性的新方法, 仿真证 明了在不同目标特性与状态工况下的方法有效性. 此外, 主惯量参数比的准确率能够达到 $90 \%$. 在所有这些工况 中, 非常重要的环节是视觉算法必须保证良好且精确的目标特征点信息. 总之, 本文给出了一种有前景与有效的 用于小天体附着前的运动与参数辨识方法, 此方法非常高效且适合星上应用.
\end{abstract}

关键词小天体探测, 非合作目标, 参数辨识, 序列图像

PACS: 05.45.-a, 95.10.Eg, 95.40.+s, 42.30.-d

\section{1 引言}

21 世纪以来, 深空小天体探测越来越受到重视, 小 天体上蕴含着丰富的稀有矿物资源，保留着太阳系形 成初期的原始成分. 科学家普遍认为, 小天体是研究 太阳系起源与演化、生命起源等问题的活化石，因此 小天体探测是当前空间探索的前沿、热点问题 ${ }^{[1]}$. 美 国、日本、欧洲都完成过或正在进行小天体探测活 动, 美国的“尼尔号”探测器对小天体“爱神”实现了成 功附着, “欧西里斯号”探测器正对小天体“贝努”进行
采样返回探测; 日本的“隼鸟I号”“隼鸟II号”探测器分 别对小天体“丝川”“龙宫”开展取样返回探测; 欧洲航 天局的“罗塞塔号”探测器首次完成了对慧星67P的附 着探测等 ${ }^{[2]}$. 我国也将积极开展小天体探测任务, 相比 于发达国家的小天体探测技术，我国的技术水平稍显 落后，尚需突破许多基础性理论与关键技术，并开展 相应的在轨试验验证. 其中需要重点突破的技术之一 就是小天体自主相对导航技术，小天体属于典型的无 标识、应答、无先验知识的空间非合作目标，对空间 非合作目标的运动状态与惯性参数辨识方法研究是小

引用格式: 邓润然, 葛东明, 史纪金金, 等. 基于序列图像的深空小天体运动状态估计. 中国科学: 物理学 力学 天文学, 2022, 52: 214511 Deng R R, Ge D M, Shi J X, et al. Estimation of motion state for small celestial body detection based on sequence images (in Chinese). Sci Sin-Phys Mech Astron, 2022, 52: 214511, doi: 10.1360/SSPMA-2021-0002 
天体相对导航的重要研究内容 ${ }^{[3-5]}$. 国内外学者对空间 目标的运动估计与惯性参数辨识开展了许多工作. Aghili和Parsa ${ }^{[6]}$ 以四元数的描述方式建立的目标状态 方程与观测方程, 提出一种自适应卡尔曼滤波方法, 实 现了目标的运动速度与测量噪声的估计, 并开展了相 应的试验验证. Shtark和Gurfii ${ }^{[7]}$ 基于立体视觉图像作 为测量输入, 研究了一种用于非合作目标识别与相对 位姿估计实时解算方法. Hou等人 ${ }^{[8]}$ 提出基于对偶向 量四元数的扩展卡尔曼滤波方法, 基于扩展卡尔曼滤 波估计了空间翻滚目标的运动参数. $\mathrm{Ma}$ 等人 ${ }^{[9]}$ 获得了 翻滚对称目标的四元数微分方程解析解, 提出了一种 扩展卡尔曼滤波方法用来实现惯性参数估计及姿态预 测. Wang等人 ${ }^{[10]}$ 研究了一种自适应不确定卡尔曼滤波 方法, 实现了空间非合作航天器的相对状态与惯量比 估计, $\mathrm{Ge}$ 等 $^{[11]}$ 仅利用立体视觉测量, 选择目标上三 个非共线特征点作为测量, 设计滤波方法实现了目标 姿态、角速度、惯量参数等的直接估计, 并通过实验 验证了滤波估计方法的收敛性与有效性.

本文研究了一种仅基于立体视觉的解算空间非合 作目标相对姿态、角速度和惯量比的快速方法. 该方 法以立体视觉获取的序列图像作为输入, 首先解算空 间非合作目标的运动状态, 然后通过不变平面与能量 球推导得到角动量与惯量比以及主惯量比, 最后通过 仿真验证了在考虑噪声的因素下快速辨识参数的有 效性.

\section{2 目标观测原理}

对目标采用双目立体视觉观测, 利用两个相机从 不同角度同时获取目标的两幅数字图像, 基于三角视 差原理恢复出目标上特征点三维位置信息的方法, 其 基本测量原理如图1所示. 图中 $o_{1}-x_{1} y_{1} z_{1}, o_{\mathrm{r}}-x_{\mathrm{r}} y_{\mathrm{r}} z_{\mathrm{r}}$ 分别 表示左、右相机坐标系, $O_{1}-X_{1} Y_{1}, O_{\mathrm{r}}-X_{\mathrm{r}} Y_{\mathrm{r}}$ 分别表示左、 右像平面坐标系 ${ }^{[12]}$.

设特征点 $T$ 在左右像平面中的位置分别为 $\left(u_{1}, v_{1}\right)$, $\left(u_{\mathrm{r}}, v_{\mathrm{r}}\right)$, 综合考虑双目相机内外参数矩阵, 根据三角 测量原理, 由左右像平面坐标可以得到特征点的物 理空间位置. 这里选取左相机坐标系描述观测特征 点的物理空间位置, 特征点在该坐标系下的坐标可 以写为

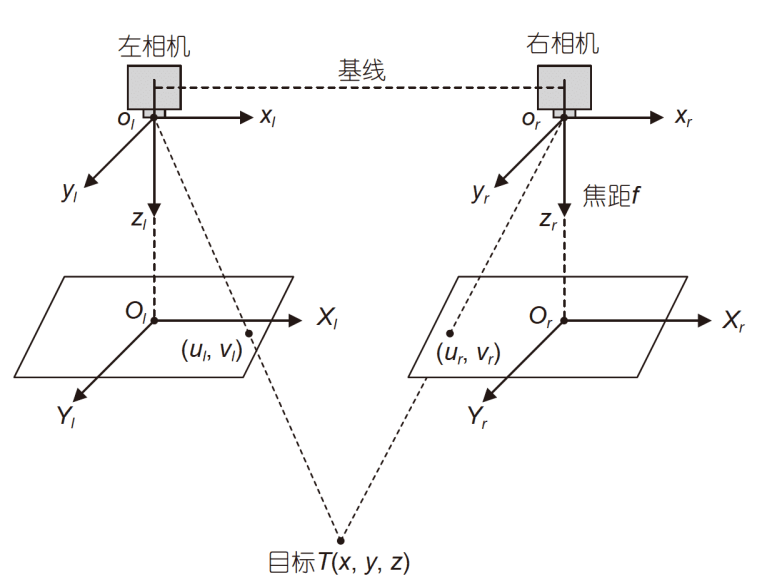

图 1 双目视觉测量原理

Figure 1 Measurement principle of binocular vision.

$$
\left\{\begin{array}{l}
x=\frac{Z_{c} \cdot a_{1}}{f_{\mathrm{lx}}}, \\
y=\frac{Z_{c} \cdot b_{1}}{f_{\mathrm{ly}}}, \\
z=\frac{f_{\mathrm{l} x} f_{\mathrm{ly}}\left(f_{\mathrm{r} x} t_{x}-a_{\mathrm{r}} t_{z}\right)}{a_{\mathrm{r}}\left(f_{\mathrm{ly}} r_{7} a_{1}+f_{\mathrm{lx}} r_{8} b_{1}+r_{9} f_{\mathrm{lx}} f_{\mathrm{ly}}\right)-f_{\mathrm{rx}}\left(f_{\mathrm{ly}} r_{1} a_{l}+f_{\mathrm{lx}} r_{2} b_{1}+r_{3} f_{\mathrm{lx}} f_{\mathrm{ly}}\right)},
\end{array}\right.
$$

其中,

$\left[\begin{array}{ccc}f_{1 x} & 0 & c_{1 x} \\ 0 & f_{1 y} & c_{1 y} \\ 0 & 0 & 1\end{array}\right]$ 为左目相机内参数矩阵; $\left[\begin{array}{ccc}f_{\mathrm{r} x} & 0 & c_{\mathrm{r} x} \\ 0 & f_{\mathrm{r} y} & c_{\mathrm{r} y} \\ 0 & 0 & 1\end{array}\right]$ 为右 目相机内参数矩阵; $\left[\begin{array}{llll}r_{1} & r_{2} & r_{3} & t_{x} \\ r_{4} & r_{5} & r_{6} & t_{y} \\ r_{7} & r_{8} & r_{9} & t_{z}\end{array}\right]$ 为相机外参数矩阵;

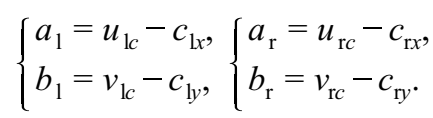

通过观测获得目标上两个固定特征点的空间位 置, 分别记为 $\left(\begin{array}{lll}x_{1} & y_{1} & z_{1}\end{array}\right),\left(\begin{array}{lll}x_{2} & y_{2} & z_{2}\end{array}\right)$, 即可获得目标上 固定矢量 $\mathbf{r}$ 相对相机坐标系的投影 $\mathbf{r}_{a}$ :

$$
\mathbf{r}_{a}=\left[\begin{array}{c}
x_{1}-x_{2} \\
y_{1}-y_{2} \\
z_{1}-z_{2}
\end{array}\right]
$$

\section{3 目标状态解算}

\section{1 姿态矩阵求解}

对于目标上一固定矢量 $\mathbf{r}$, 它在由不同基矢量组成 
的参考系 $\left\{\mathrm{F}_{a}\right\},\left\{\mathrm{F}_{b}\right\}$ 中分别有投影 $\mathbf{r}_{a}, \mathbf{r}_{b}$ :

$\mathrm{F}_{a}=\left[\begin{array}{c}\overrightarrow{\mathbf{a}}_{1} \\ \mathbf{a}_{2} \\ \overrightarrow{\mathbf{a}}_{3}\end{array}\right], \mathrm{F}_{b}=\left[\begin{array}{c}\overrightarrow{\mathbf{b}}_{1} \\ \overrightarrow{\mathbf{b}}_{2} \\ \overrightarrow{\mathbf{b}}_{3}\end{array}\right], \mathbf{r}_{a}=\left[\begin{array}{c}r_{a 1} \\ r_{a 2} \\ r_{a 3}\end{array}\right], \mathbf{r}_{b}=\left[\begin{array}{c}r_{b 1} \\ r_{b 2} \\ r_{b 3}\end{array}\right]$,

那么有如下表达式:

$\mathbf{r}=\mathbf{r}_{a}^{\mathrm{T}} \mathrm{F}_{a}=\mathbf{r}_{b}^{\mathrm{T}} \mathrm{F}_{b}, \overrightarrow{\mathbf{r}}=\mathrm{F}_{a}^{\mathrm{T}} \mathbf{r}_{a}=\mathrm{F}_{b}^{\mathrm{T}} \mathbf{r}_{b}$,

所以

$\mathbf{r}_{a}^{\mathrm{T}}=\mathbf{r}_{b}^{\mathrm{T}} \mathrm{F}_{b} \cdot \mathrm{F}_{a}{ }^{\mathrm{T}}=\mathbf{r}_{b}^{\mathrm{T}} \mathbf{C}_{b a}$,

其中, $\mathbf{C}_{b a}=\mathrm{F}_{b} \cdot \mathrm{F}_{a}{ }^{\mathrm{T}}$ 即为方向余弦矩阵, 即正交单位 矩阵.

设 $\left\{\mathrm{F}_{a}\right\}$ 为惯性系, $\left\{\mathrm{F}_{b}\right\}$ 为空间非合作目标固连系, 对于初始时刻, $\left\{\mathrm{F}_{b}\right\}$ 与 $\left\{\mathrm{F}_{a}\right\}$ 平行. 对于空间非合作目标 固连系上一固定矢量 $\overrightarrow{\mathbf{r}}_{b}$, 初始时刻其在 $\left\{\mathrm{F}_{b}\right\}$ 与 $\left\{\mathrm{F}_{a}\right\}$ 的投 影为 $\mathbf{r}_{b}=\mathbf{r}_{a, 0} t$ 时刻其在 $\left\{\mathrm{F}_{b}\right\}$ 与 $\left\{\mathrm{F}_{a}\right\}$ 的投影为 $\mathbf{r}_{b}=\mathbf{C}_{b a, t} \mathbf{r}_{a, t}$.

因此，获得目标上三个固定的非共线特征点 $P_{1}$, $P_{2}, P_{3}$, 如图2所示.

三个特征点可以建立两个空间非合作目标上固连 矢量 $\overrightarrow{\mathbf{r}}_{b 1}, \overrightarrow{\mathbf{r}}_{b 2}$, 这两个矢量初始时刻在惯性系的观测投影 为 $\mathbf{r}_{a 1,0}, \mathbf{r}_{a 2,0}$, 与t时刻在惯性系的观测投影为 $\mathbf{r}_{a 1, t}, \mathbf{r}_{a 2, t}$, 可 以获得姿态矩阵 $\mathbf{C}_{b a, t}$ :

$\mathbf{C}_{b a, t}=\left[\begin{array}{lll}\mathbf{r}_{a 1,0} & \mathbf{r}_{a 2,0} & \mathbf{r}_{a 1,0} \times \mathbf{r}_{a 2,0}\end{array}\right] \cdot\left[\begin{array}{lll}\mathbf{r}_{a 1, t} & \mathbf{r}_{a 2, t} & \mathbf{r}_{a 1, t} \times \mathbf{r}_{a 2, t}\end{array}\right]^{-1}$.

\section{2 角速度求解}

采用(·)表示在参考系 $\left\{\mathrm{F}_{a}\right\}$ 中观察矢量对时间的变 化率, 用(o)表示在 $\left\{\mathrm{F}_{b}\right\}$ 中观察矢量对时间的变化率, 因此,

$\dot{\mathrm{F}}_{a} \equiv 0 ; \stackrel{\circ}{\mathrm{F}}_{b} \equiv 0$.

对于坐标系 $\left\{\mathrm{F}_{b}\right\}$ 的基矢量相对 $\left\{\mathrm{F}_{a}\right\}$ 的变化存在对 时间导数为

$\dot{\mathbf{b}}_{1}=\overrightarrow{\boldsymbol{\omega}}_{b a} \times \overrightarrow{\mathbf{b}}_{1} ; \dot{\mathbf{b}}_{2}=\overrightarrow{\boldsymbol{\omega}}_{b a} \times \overrightarrow{\mathbf{b}}_{2} ; \dot{\mathbf{b}}_{3}=\overrightarrow{\boldsymbol{\omega}}_{b a} \times \overrightarrow{\mathbf{b}}_{3} ;$

所以

$\dot{\mathrm{F}}_{b}=\overrightarrow{\boldsymbol{\omega}}_{b a} \times \mathrm{F}_{b}$.

对姿态旋转矩阵 $\mathbf{C}_{b a}$ 的时间导数如下:



图 2 目标特征点示意图

Figure 2 Feature points on the target.

$\frac{\mathrm{d} \mathbf{C}_{b a}}{\mathrm{~d} t}=\frac{\mathrm{d}\left(\mathrm{F}_{b} \cdot \mathrm{F}_{a}^{\mathrm{T}}\right)}{\mathrm{d} t}=\dot{\mathrm{F}}_{b} \cdot \mathrm{F}_{a}^{\mathrm{T}}+\mathrm{F}_{b} \cdot \dot{\mathrm{F}}_{a}$,

都在 $\left\{\mathrm{F}_{a}\right\}$ 系中观察各坐标系的变化, 在 $\left\{\mathrm{F}_{a}\right\}$ 系中观察 $\mathrm{F}_{a}$ 是不变的, 即 $\dot{\mathrm{F}}_{a}=0$, 那么

$$
\begin{aligned}
\frac{\mathrm{d} \mathbf{C}_{b a}}{\mathrm{~d} t} & =\dot{F}_{b} \cdot \mathrm{F}_{a}^{\mathrm{T}} \\
& =\left(\overrightarrow{\boldsymbol{\omega}}_{b a} \times \mathrm{F}_{b}\right) \cdot \mathrm{F}_{a}^{\mathrm{T}} \\
& =\left(\vec{\omega}_{b a} \times \mathrm{F}_{b}^{\mathrm{T}}\right)^{\mathrm{T}} \cdot \mathrm{F}_{a}^{\mathrm{T}} \\
& =\left(\boldsymbol{\omega}_{b a}^{\mathrm{T}} \mathrm{F}_{b} \times \mathrm{F}_{b}^{\mathrm{T}}\right)^{\mathrm{T}} \cdot \mathrm{F}_{a}^{\mathrm{T}} \\
& =\left(\mathrm{F}_{b}^{\mathrm{T}} \boldsymbol{\omega}_{b a}^{\times}\right)^{\mathrm{T}} \cdot \mathrm{F}_{a}^{\mathrm{T}} \\
& =-\boldsymbol{\omega}_{b a}^{\times} \mathrm{F}_{b} \cdot \mathrm{F}_{a}^{\mathrm{T}} \\
& =-\boldsymbol{\omega}_{b a}^{\times} C_{b a},
\end{aligned}
$$

即角速度有

$\boldsymbol{\omega}_{b a}^{\times}=-\dot{\mathbf{C}}_{b a} \mathbf{C}_{b a}^{\mathrm{T}}$,

其中, $\boldsymbol{\omega}_{b a}^{\times}=\left[\begin{array}{ccc}0 & -\omega_{b a 3} & \omega_{b a 2} \\ \omega_{b a 3} & 0 & -\omega_{b a 1} \\ -\omega_{b a 2} & \omega_{b a 1} & 0\end{array}\right]$.

至此, 只需获取了空间非合作目标与观测航天器 的时变姿态矩阵, 即可进一步获得空间非合作目标瞬 时角速度 $\boldsymbol{\omega}_{b a}$ 在连体系 $\left\{\mathrm{F}_{b}\right\}$ 的投影.

\section{3 角动量比与惯量比}

对于自由运动空间非合作目标，其角动量与系统 能量是守恒的. 设空间非合作目标系统能量为 $T$ 、角 
动量为 $\overrightarrow{\mathbf{H}}$ 、角速度为 $\boldsymbol{\omega}$, 惯量为 $\overrightarrow{\mathbf{J}}, \overrightarrow{\mathbf{H}}$ 与 $\boldsymbol{\boldsymbol { \omega }}$ 夹角为 $\theta$, 角动 量、角速度和惯量在惯性系 $\left\{\mathrm{F}_{a}\right\}$ 与连体坐标系 $\left\{\mathrm{F}_{b}\right\}$ 下 的描述分别为 $\mathbf{H}_{a}, \mathbf{H}_{b}, \boldsymbol{\omega}_{a}, \boldsymbol{\omega}_{b}, \mathbf{J}_{a}, \mathbf{J}_{b}$, 且 $\left\{\mathrm{F}_{b}\right\}$ 原点在空间 非合作目标质心, 系统能量与角动量如下表达:

$2 T=\boldsymbol{\omega} \cdot \overrightarrow{\mathbf{J}} \cdot \boldsymbol{\omega}$,

$\vec{H}=\mathbf{J} \cdot \boldsymbol{\omega}$,

其中, 能量与角动量在惯性系 $\left\{\mathrm{F}_{a}\right\}$ 与连体坐标系 $\left\{\mathrm{F}_{b}\right\}$ 下 的标量表达式为

$$
\begin{aligned}
& T=\frac{\boldsymbol{\omega}_{a}^{\mathrm{T}} \mathbf{J}_{a} \boldsymbol{\omega}_{a}}{2}=\frac{\boldsymbol{\omega}_{b}^{\mathrm{T}} \mathbf{J}_{b} \boldsymbol{\omega}_{b}}{2}, \\
& \mathbf{H}_{a}=\mathbf{J}_{a} \boldsymbol{\omega}_{a}=\left[\begin{array}{lll}
H_{a x} & H_{a y} & H_{a z}
\end{array}\right]^{\mathrm{T}}, \\
& \mathbf{H}_{b}=\mathbf{J}_{b} \boldsymbol{\omega}_{b}=\mathbf{C}_{b a} \mathbf{J}_{a} \mathbf{C}_{b a}^{\mathrm{T}} \mathbf{C}_{b a} \boldsymbol{\omega}_{a}=\mathbf{C}_{b a} \mathbf{H}_{a},
\end{aligned}
$$

那么

$2 T=\overrightarrow{\boldsymbol{\omega}} \cdot \mathbf{H}$,

$|\boldsymbol{\omega}| \cdot \cos \theta=\frac{2 T}{|\mathbf{H}|}=$ const.

因为空间非合作目标系统角动量 $\mathbf{H}$ 是守恒的，由 式(19)可知，空间非合作目标瞬时角速度 $\boldsymbol{\omega}$ 矢量沿 $\mathbf{H}$ 方 向的分量是常值，即终点在将落在同一平面上，平面 法向即为 $\mathbf{H}$ 方向. 因此, 在惯性系 $\left\{\mathrm{F}_{a}\right\}$ 中观察角速度, $\boldsymbol{\omega}_{a}$ 元素坐标在同一平面内.

前述已经获得角速度在空间非合作目标连体 系 $\left\{\mathrm{F}_{b}\right\}$ 的投影 $\boldsymbol{\omega}_{b}$, 以及惯性系 $\left\{\mathrm{F}_{a}\right\}$ 与连体坐标系 $\left\{\mathrm{F}_{b}\right\}$ 的 姿态旋转矩阵 $\mathbf{C}_{b a}$, 所以角速度在惯性系 $\left\{\mathrm{F}_{b}\right\}$ 的投影 $\boldsymbol{\omega}_{a}$ 也是已知的:

$\boldsymbol{\omega}_{a}=\mathbf{C}_{b a}^{\mathrm{T}} \boldsymbol{\omega}_{b}$.

通过对角速度 $\boldsymbol{\omega}_{a}$ 进行平面拟合，即可获得空间非 合作目标系统角动量 $\mathbf{H}$ 的指向，其在惯性系的投影 即为 $\hat{\mathbf{H}}_{a}$ :

$$
\begin{aligned}
\widehat{\mathbf{H}}_{a} & =\frac{\mathbf{H}_{a}}{H_{a z}}=\left[\begin{array}{lll}
\frac{H_{a x}}{H_{a z}} & \frac{H_{a y}}{H_{a z}} & 1
\end{array}\right]^{\mathrm{T}} . \\
& \text { 已知 } \\
\mathbf{J}_{b} \boldsymbol{\omega}_{b} & =\mathbf{C}_{b a} \mathbf{H}_{a},
\end{aligned}
$$

那么
$\widehat{\mathbf{J}}_{b} \boldsymbol{\omega}_{b}=\mathbf{C}_{b a} \widehat{\mathbf{H}}_{a}$

其中, $\widehat{\mathbf{J}}_{b}=\frac{\mathbf{J}_{b}}{H_{a z}}$.

对空间非合作目标观测一定时间，获得三个时刻 的本体角速度为 $\boldsymbol{\omega}_{b, t 1}, \boldsymbol{\omega}_{b, 22}, \boldsymbol{\omega}_{b, t 3}$, 对应的空间非合作目 标连体系到惯性系的姿态旋转矩阵为 $\mathbf{C}_{b a, 1}, \mathbf{C}_{b a, t 2}, \mathbf{C}_{b a, t 3}$, 那么可得下式:

$\widehat{\mathbf{J}}_{b}\left[\begin{array}{lll}\boldsymbol{\omega}_{b, t 1} & \boldsymbol{\omega}_{b, t 3} & \boldsymbol{\omega}_{b, t 3}\end{array}\right]=\left[\begin{array}{lll}\mathbf{C}_{b a, t 1} \widehat{\mathbf{H}}_{a} & \mathbf{C}_{b a, t 2} \widehat{\mathbf{H}}_{a} & \mathbf{C}_{b a, t 3} \widehat{\mathbf{H}}_{a}\end{array}\right]$,

进一步求解得

$$
\widehat{\mathbf{J}}_{b}=\left[\begin{array}{llll}
\mathbf{C}_{b a, t 1} \widehat{\mathbf{H}}_{a} & \mathbf{C}_{b a, t 2} \widehat{\mathbf{H}}_{a} & \mathbf{C}_{b a, t 3} \widehat{\mathbf{H}}_{a}
\end{array}\right] \cdot\left[\begin{array}{lll}
\boldsymbol{\omega}_{b, t 1} & \boldsymbol{\omega}_{b, t 3} & \boldsymbol{\omega}_{b, t 3}
\end{array}\right]^{-1} .
$$

至此, 即获得了空间非合作目标的惯量比矩阵 $\mathbf{J}_{b}$ :

$$
\widehat{\mathbf{J}}_{b}=\left[\begin{array}{lll}
\widehat{\mathbf{J}}_{b, 11} & \widehat{\mathbf{J}}_{b, 12} & \widehat{\mathbf{J}}_{b, 13} \\
\widehat{\mathbf{J}}_{b, 12} & \widehat{\mathbf{J}}_{b, 22} & \widehat{\mathbf{J}}_{b, 23} \\
\widehat{\mathbf{J}}_{b, 13} & \widehat{\mathbf{J}}_{b, 23} & \widehat{\mathbf{J}}_{b, 33}
\end{array}\right] .
$$

可以按 $\mathbf{J}_{b, 33}$ 进一步归一化得

$\widehat{\mathbf{J}}_{b}=\frac{\widehat{\mathbf{J}}_{b}}{\widehat{\mathbf{J}}_{b, 33}}=\left[\begin{array}{ccc}\widehat{\mathbf{J}}_{b, 11} & \widehat{\mathbf{J}}_{b, 12} & \widehat{\mathbf{J}}_{b, 13} \\ \widehat{\mathbf{J}}_{b, 12} & \widehat{\mathbf{J}}_{b, 22} & \widehat{\mathbf{J}}_{b, 23} \\ \widehat{\mathbf{J}}_{b, 13} & \widehat{\mathbf{J}}_{b, 23} & 1\end{array}\right]$.

可进一步对 $\widehat{\mathbf{J}}_{b}$ 分解, 获得目标主轴方向惯量比 $\widehat{\mathbf{J}}_{A}$ :

$\widehat{\mathbf{J}}_{\Lambda}=\mathbf{C}_{\Lambda b} \widehat{\mathbf{J}}_{b} \mathbf{C}_{\Lambda b}^{\mathrm{T}}=\left[\begin{array}{lll}\widehat{\mathbf{J}}_{\Lambda, 1} & & \\ & \widehat{\mathbf{J}}_{\Lambda, 2} & \\ & & \widehat{\mathbf{J}}_{\Lambda, 3}\end{array}\right]$,

其中 $\mathbf{C}_{A b}$ 为空间非合作目标本体坐标系到主轴系的姿 态旋转矩阵. 以上仅采用了三个时刻的输入获得了一 次解算结果, 可进一步选择多组输入多次解算, 采用 优化方法获得最优的解算结果, 本文不再赘述.

\section{4 仿真算例分析}

\section{1 动力学模型与参数}

假定一空间目标航天器无外力作用下自由飞行, 建立系统相对本体坐标系下的单刚体动力学模型 
如下:

$$
\left\{\begin{array}{l}
m \dot{\mathbf{v}}_{b}-\widetilde{\mathbf{c}}_{b} \dot{\boldsymbol{\omega}}_{b}+\widetilde{\boldsymbol{\omega}}_{b} m \mathbf{v}_{b}-\widetilde{\boldsymbol{\omega}}_{b} \widetilde{\mathbf{c}}_{b} \boldsymbol{\omega}_{b}=0, \\
\widetilde{\mathbf{c}}_{b} \dot{\mathbf{v}}_{b}+\mathbf{J}_{b} \dot{\boldsymbol{\omega}}_{b}-\widetilde{\mathbf{v}}_{b} \widetilde{\mathbf{c}}_{b} \boldsymbol{\omega}_{b}+\widetilde{\boldsymbol{\omega}}_{b} \widetilde{\mathbf{c}}_{b} \mathbf{v}_{b}+\widetilde{\boldsymbol{\omega}}_{b} \mathbf{J}_{b} \boldsymbol{\omega}_{b}=0,
\end{array}\right.
$$

其中, $m$ 为系统质量; $\mathbf{c}_{b}$ 为系统静矩; $\mathbf{J}_{b}$ 为系统惯性矩; $\mathbf{v}_{b}$ 为平动速度; $\boldsymbol{\omega}_{b}$ 为转动速度. 当本体坐标系原点取在 航天器质心时, 上式化简为

$$
\left[\begin{array}{cc}
m & 0 \\
0 & \mathbf{J}_{b}
\end{array}\right]\left[\begin{array}{c}
\dot{\mathbf{v}}_{b} \\
\dot{\boldsymbol{\omega}}_{b}
\end{array}\right]+\left[\begin{array}{cc}
\widetilde{\boldsymbol{\omega}}_{b} & 0 \\
0 & \widetilde{\boldsymbol{\omega}}_{b}
\end{array}\right]\left[\begin{array}{cc}
m & 0 \\
0 & \mathbf{J}_{b}
\end{array}\right]\left[\begin{array}{c}
\mathbf{v}_{b} \\
\boldsymbol{\omega}_{b}
\end{array}\right]=0 .
$$

取系统质量为 $3000 \mathrm{~kg}$, 在目标一连体坐标系下描 述其相对质心转动惯量为

$$
\mathbf{J}_{b}=\left[\begin{array}{ccc}
5887.73 & -970.31 & -3193.43 \\
-970.31 & 12708.29 & -1063.51 \\
-3193.43 & -1063.51 & 9883.97
\end{array}\right]\left(\mathrm{kg} \mathrm{m}^{2}\right) \text {. }
$$

目标初始旋转角速度:

$$
\boldsymbol{\omega}_{b 0}=\left[\begin{array}{lll}
1 & 0.2 & 0.1
\end{array}\right]^{\mathrm{T}}(\%) \text {. }
$$

航天器惯量比矩阵在连体系下描述为

$$
\widehat{\mathbf{J}}_{b}=\left[\begin{array}{ccc}
0.5957 & -0.0982 & -0.3231 \\
-0.0982 & 1.2857 & -0.1076 \\
-0.3231 & -0.1076 & 1
\end{array}\right] \text {. }
$$

航天器主轴惯量比矩阵在连体系下描述为

$$
\widehat{\mathbf{J}}_{A}=\left[\begin{array}{lll}
1.3219 & & \\
& 1.1642 & \\
& & 0.3953
\end{array}\right] \text {. }
$$

设惯性系与空间非合作目标连体系初始时刻平 行, 系统角动量为

$$
\mathbf{H}_{a}=\left[\begin{array}{lll}
93.7997 & 25.5691 & -42.1975
\end{array}\right]^{\mathrm{T}}(\mathrm{N} \mathrm{m} \mathrm{s}) \text {. }
$$

按 Z元素归一化的系统角动量比为

$$
\widehat{\mathbf{H}}_{a}=\left[\begin{array}{lll}
-2.2229 & -0.6059 & 1
\end{array}\right]^{\mathrm{T}} \text {. }
$$

\section{2 不考虑噪声的辨识分析}

空间非合作目标自由滚动, 选择目标连体系下两 矢量如下:

$$
\begin{aligned}
& \mathbf{r}_{b 1}=\left[\begin{array}{lll}
1 & 1 & 3
\end{array}\right]^{\mathrm{T}}(\mathrm{m}), \\
& \mathbf{r}_{b 2}=\left[\begin{array}{lll}
3 & 1 & 3
\end{array}\right]^{\mathrm{T}}(\mathrm{m}) .
\end{aligned}
$$

观测目标连体坐标系下两个矢量的在惯性系的投 影, 通过动力学仿真得到两个矢量各时刻在惯性系的
坐标 $\mathbf{r}_{a 1}, \mathbf{r}_{a 2}$, 作为辨识解算的不考虑噪声的已知输入, 按照本文的方法, 辨识空间非合作目标角速度在连体 系的投影与动力学模型仿真角速度对比, 如图3所示. 蓝色曲线表示通过辨识得到的角速度, 红色曲线代表 通过仿真得到的角速度, 两条曲线完全吻合.

通过辨识的姿态转换矩阵将角速度换算到惯性 系，与动力学模型仿真计算得到的惯性系角速度对比 如图4. 蓝色曲线表示通过辨识处理的结果, 红色曲线 代表通过仿真得到的结果, 两条曲线完全吻合.

辨识空间非合作目标角速度在惯性系与连体系下 轨迹如图5 和6.

可见，空间非合作目标角速度在惯性系的投影轨
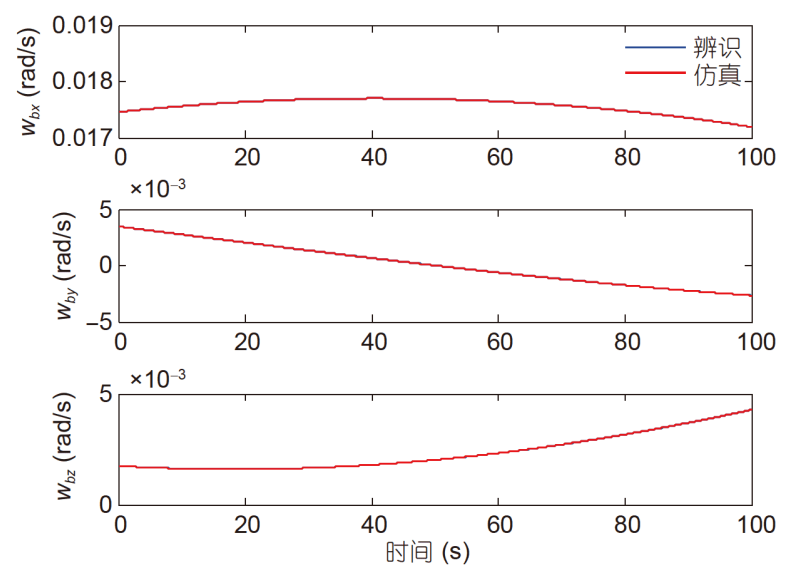

图 3 (网络版彩图)连体系下角速度对比

Figure 3 (Color online) Contrast of angular velocities on the bodyfixed coordinate system.
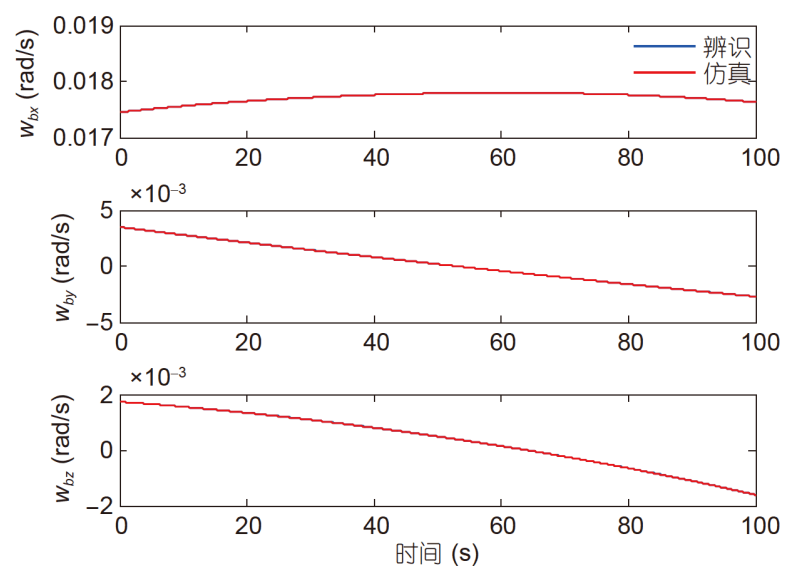

图 4 (网络版彩图)惯性系下角速度对比

Figure 4 (Color online) Contrast of angular velocities on the absolute coordinate system. 


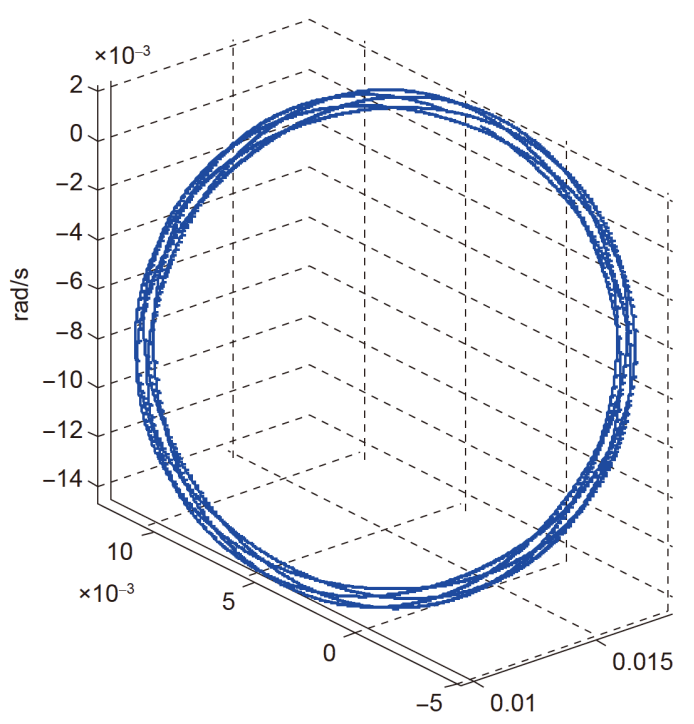

图 5 (网络版彩图) 惯性系下角速度轨迹

Figure 5 (Color online) Trajectory of angular velocity on the absolute coordinate system.

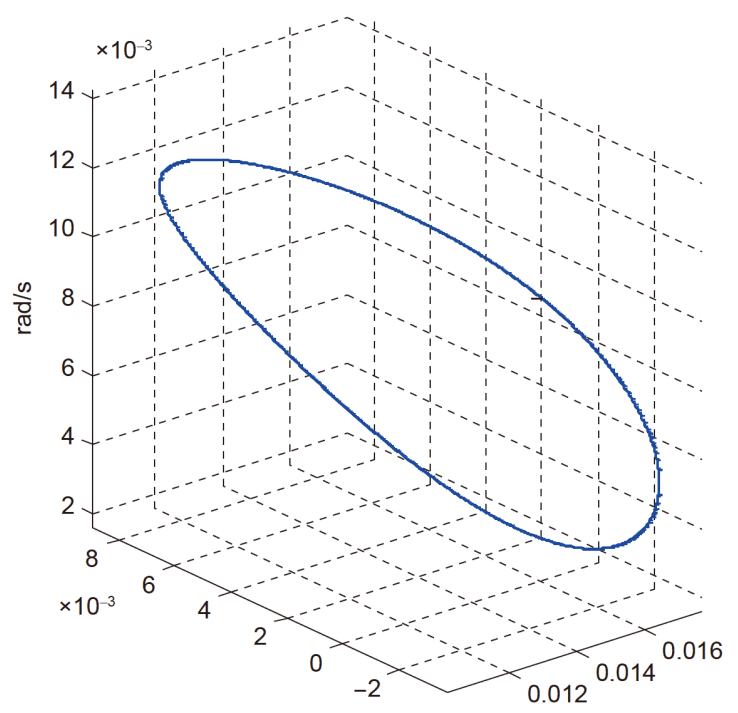

图 6 (网络版彩图)连体系下角速度

Figure 6 (Color online) Trajectory of angular velocity on the bodyfixed coordinate system.

迹在一个平面内，角速度在连体系的投影轨迹为一闭 合空间曲线，实际是椭圆上的一条迹线. 通过对惯性 系下角速度轨迹拟合平面，获得平面法向量，即为识 别的角动量方向, 得到按Z元素归一化的角动量比:

solve_$\widehat{H}_{a}=\left[\begin{array}{lll}-2.2205 & -0.6058 & 1\end{array}\right]^{\mathrm{T}}$.
角动量比识别误差为

error_ $\widehat{\mathbf{H}}_{a}=\left|\frac{\widehat{\mathbf{H}}_{a}-\text { solve_} \widehat{\mathbf{H}}_{a}}{\widehat{\mathbf{H}}_{a}}\right|=\left[\begin{array}{lll}0.11 \% & 0.03 \% & 0\end{array}\right]^{\mathrm{T}}$.

仅选择1，5，10 s三个时刻，按照本文方法辨识空 间非合作目标相对连体系惯量比与主轴系惯量比为

$$
\begin{aligned}
& \text { solve_ } \widehat{\mathbf{J}}_{b}=\left[\begin{array}{ccc}
0.5948 & -0.0981 & -0.3230 \\
-0.0981 & 1.2849 & -0.1082 \\
-0.3230 & -0.1076 & 1
\end{array}\right] \text {, } \\
& \text { solve_恿 }=\left[\begin{array}{lll}
1.3214 & & \\
& 1.1637 & \\
& & 0.3946
\end{array}\right] \text {. }
\end{aligned}
$$

航天器相对连体系惯量比, 主惯量比识别误差为

$$
\begin{aligned}
& \text { error_ } \widehat{\mathbf{J}}_{b}=\left[\begin{array}{ccc}
0.15 \% & 0.12 \% & 0.02 \% \\
0.03 \% & 0.07 \% & 0.55 \% \\
0.04 \% & 0.05 \% & 0
\end{array}\right] \text {, } \\
& \text { error_踣 }=\left[\begin{array}{lll}
0.04 \% & & \\
& 0.05 \% & \\
& & 0.16 \%
\end{array}\right] \text {. }
\end{aligned}
$$

进一步可选择任意多组三个时刻, 优化得到最优识别 结果, 非本文重点不做介绍.

\section{3 考虑噪声的辨识优化分析}

仍对同一参数模型开展动力学仿真, 引入噪声项 作为观测误差, 通过滤波拟合数据优化得到辨识结果. 对观测目标连体坐标系下两个矢量 $\mathbf{r}_{b 1}, \mathbf{r}_{b 2}$ 各时刻在惯 性系的坐标 $\mathbf{r}_{a 1}, \mathbf{r}_{a 2}$, 分别引入两项高斯噪声误差 $\varepsilon_{1}, \varepsilon_{2}$, 误差均值为 0 , 方差为

$$
\begin{aligned}
& \operatorname{Var}\left(\varepsilon_{1}\right)=\left(1 \% \cdot\left|\mathbf{r}_{b 1}\right|\right)^{2}, \\
& \operatorname{Var}\left(\varepsilon_{2}\right)=\left(1 \% \cdot\left|\mathbf{r}_{b 2}\right|\right)^{2} .
\end{aligned}
$$

辨识解算的考虑噪声的观测输入为

$$
\begin{aligned}
\mathbf{r}_{a 1} & =\mathbf{r}_{a 1}+\varepsilon_{1}, \\
\mathbf{r}_{a 2} & =\mathbf{r}_{a 2}+\varepsilon_{2} .
\end{aligned}
$$

对某一时刻观测值, 求解其局部多点的平均值, 即 采用移动平均滤波器对观测的矢量投影进行平滑处 理, 获得引入噪声项的观测矢量滤波前后的变化曲线, 图7蓝色曲线为引入噪声后的 $\mathbf{r}_{a 1}$ 三个分量变化情况, 红 色曲线为对数据进行滤波后的优化结果.

通过对滤波后数据进一步开展辨识分析, 辨识空 
间非合作目标角速度在连体系的坐标, 并与动力学模 型仿真计算的理想角速度对比, 如图8所示.

通过辨识的姿态转换矩阵将角速度换算到惯性 系，与动力学模型仿真计算得到的惯性系理想角速度 对比如图9所示.

辨识并滤波后的空间非合作目标角速度在本体系 下轨迹如图10所示, 由图10可知, 角速度轨迹旋转完成 一圈, 若按照理想无噪声情况, 其轨迹结果如图6, 当本 体角速度轨迹变化达到一圈时, 目标即旋转了一个 周期.
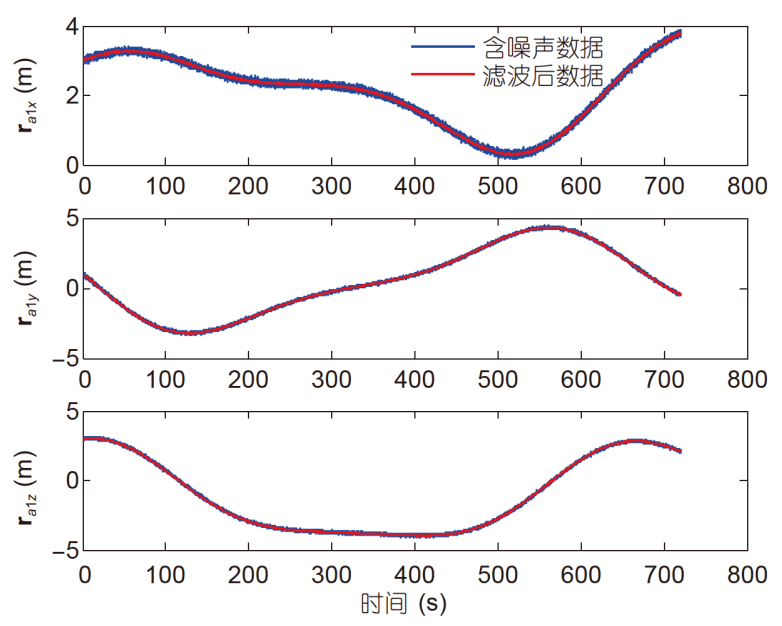

图 7 (网络版彩图)观测矢量的变化曲线

Figure 7 (Color online) Variation of vector observation with noise.


图 8 (网络版彩图)考虑噪声的连体系下角速度对比

Figure 8 (Color online) Contrast of angular velocities with noise on the body-fixed coordinate system.
辨识并滤波后的空间非合作目标角速度在惯性系 下轨迹如图11所示, 按照本文的辨识算法, 将对角速度 在惯性系下的轨迹作不变平面拟合，当本体角速度轨 迹旋转达到 $0.5-1$ 圈时，可以实现平面拟合，即目标旋 转半个到一个周期时, 可以获取辨识结果.

通过对惯性系下角速度轨迹拟合平面，获得平面 法向量, 即为识别的角动量比与识别误差为

solve_$\widehat{\mathbf{H}}_{a}=\left[\begin{array}{lll}-2.1001 & -0.5474 & 1\end{array}\right]^{\mathrm{T}}$,

error_ $\widehat{\mathbf{H}}_{a}=\left[\begin{array}{lll}5.52 \% & 9.66 \% & 0\end{array}\right]^{\mathrm{T}}$.
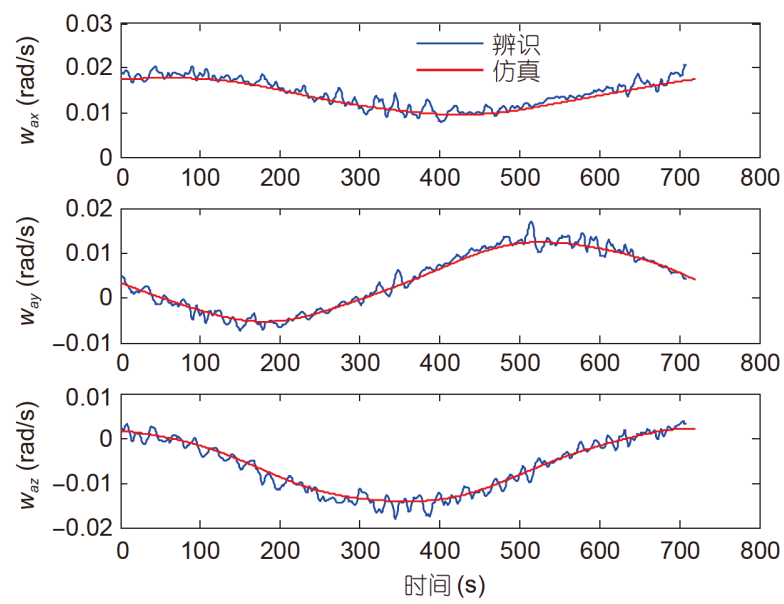

图 9 (网络版彩图)考虑噪声的惯性系下角速度对比

Figure 9 (Color online) Contrast of angular velocities with noise on the absolute coordinate system.



图 10 滤波后的本体系下角速度轨迹

Figure 10 Trajectory of angular velocity after filter on the body-fixed coordinate system. 


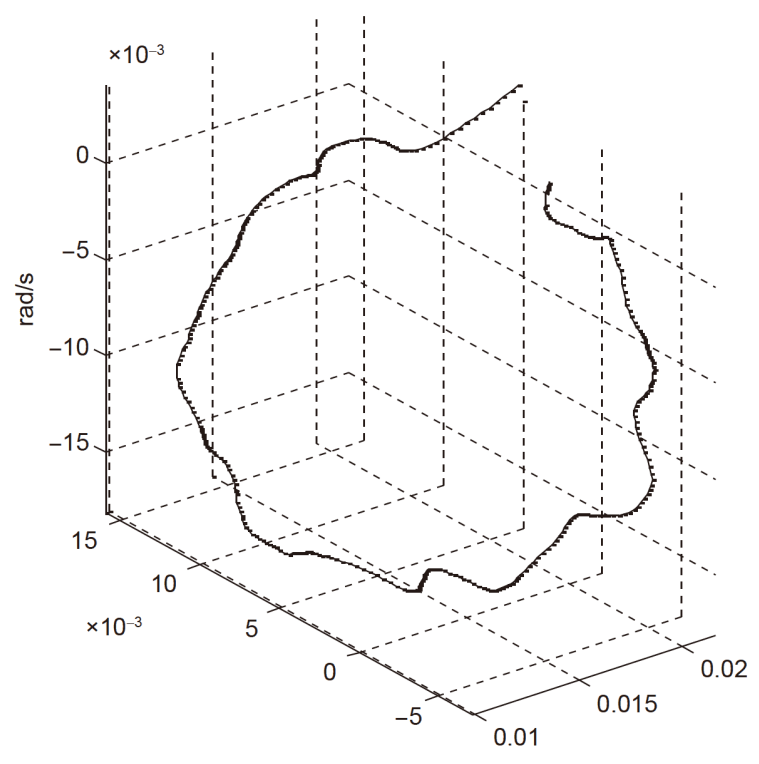

图 11 滤波后的惯性系下角速度轨迹

Figure 11 Trajectory of angular velocity after filter on the vabsolute coordinate system.

对全部仿真数据均分三段组成若干组三个时刻数 据，按照本文方法辨识空间非合作目标相对连体系惯 量比与主轴系惯量比, 并统计期望值为

solve_ $\widehat{\mathbf{J}}_{b}=\left[\begin{array}{ccc}0.5496 & -0.0921 & -0.3051 \\ -0.0921 & 1.2968 & -0.1216 \\ -0.3051 & -0.1216 & 1\end{array}\right]$,



航天器相对连体系惯量比, 主惯量比识别误差为

$$
\begin{aligned}
& \text { error_ } \widehat{\mathbf{J}}_{b}=\left[\begin{array}{ccc}
7.74 \% & 6.16 \% & 5.56 \% \\
6.16 \% & 0.86 \% & 12.97 \% \\
5.56 \% & 12.97 \% & 0
\end{array}\right] \text {, } \\
& \text { error_预}=\left[\begin{array}{lll}
1.42 \% & & \\
& 2.89 \% & \\
& & 5.12 \%
\end{array}\right] \text {. }
\end{aligned}
$$

对此算例进行 100 次考虑噪声的仿真, 重复以上分 析过程并统计辨识结果，给出角动量比的辨识误差分 布(图12)、目标连体系惯量比误差分布(图13)、目标 主轴系惯量比误差分布曲线(图14)).

100 次的目标角动量比识别误差期望为

error_ $\widehat{\mathbf{H}}_{a}=\left[\begin{array}{lll}4.51 \% & 4.17 \% & 0\end{array}\right]^{\mathrm{T}}$.


图 12 (网络版彩图)角动量比的辨识误差分布

Figure 12 (Color online) Identification error distribution of angular momentum ratios.
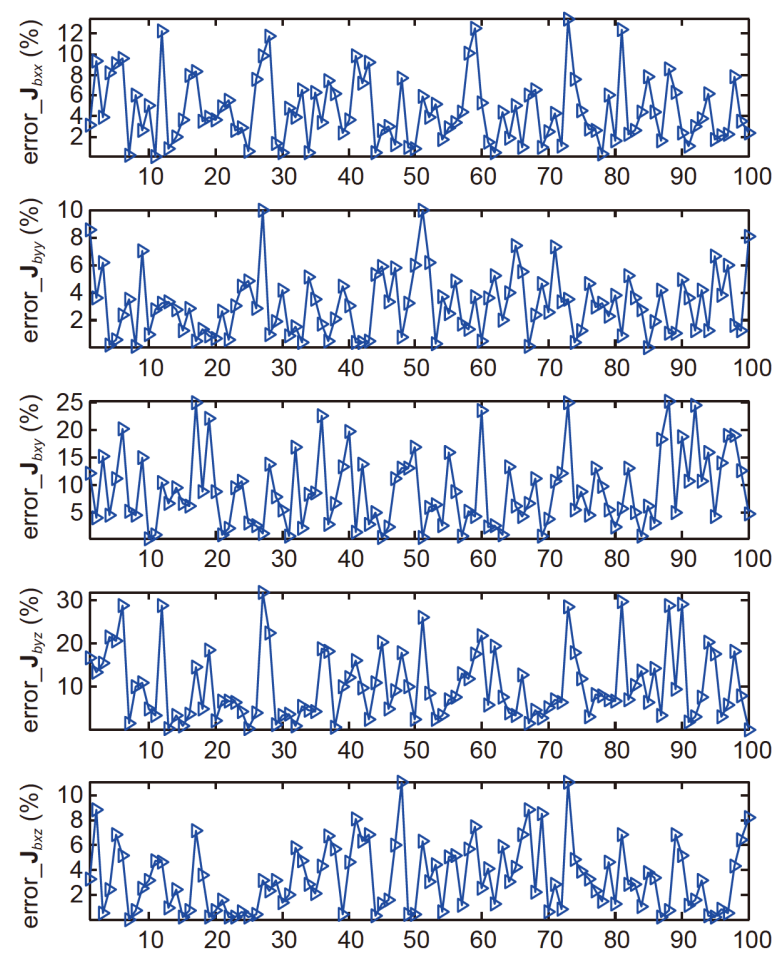

图 13 (网络版彩图)目标连体系惯量比误差分布

Figure 13 (Color online) Identification error distribution of inertia ratios on the body-fixed coordinate system.

100次的目标相对连体系惯量比识别误差期望为

error $\_\widehat{\mathbf{J}}_{b}=\left[\begin{array}{ccc}4.58 \% & 8.95 \% & 3.35 \% \\ 8.95 \% & 3.10 \% & 10.12 \% \\ 3.35 \% & 10.12 \% & 0\end{array}\right]$. 

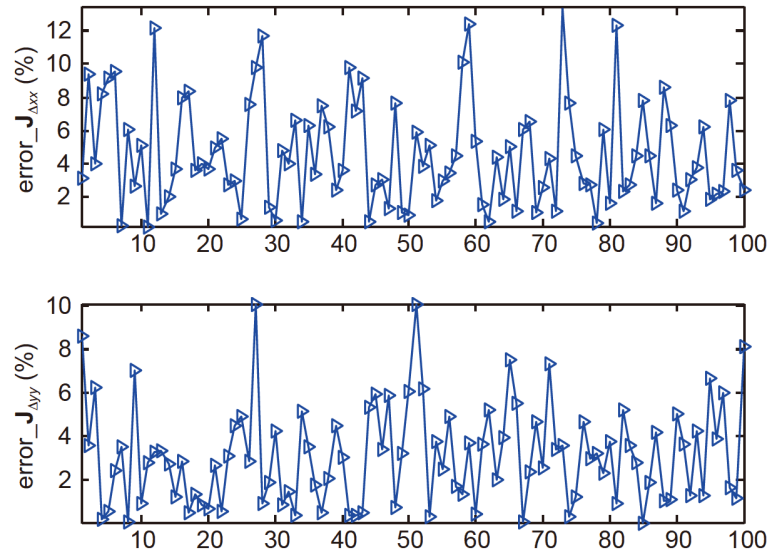

图 14 (网络版彩图)目标主轴系惯量比误差分布

Figure 14 (Color online) Identification error distribution of inertia ratios on the principal coordinate system.

100 次的目标相对主轴系惯量比识别误差期望为

$$
\text { error_预 }=\left[\begin{array}{lll}
2.97 \% & & \\
& 1.12 \% & \\
& & 3.37 \%
\end{array}\right] \text {. }
$$

\section{4 模拟小天体算例仿真分析}

本节以小天体 $2016 \mathrm{HO}_{3}$ 的物理参数为参考, 开展 仿真算例分析验证. $2016 \mathrm{HO}_{3}$ 为近年来来新发现的地 球准卫星小天体 ${ }^{[13]}$, 根据已知观测参数, 该小天体直 径约 $40 \mathrm{~m}$, 自转周期约 $0.467 \mathrm{~h}$, 自转角速度约 $0.21 \% \mathrm{~s}$, 按照该参数设计近似算例 ${ }^{[14]}$.

假定一小天体质量为 $2512 \mathrm{t}$, 密度 $2000 \mathrm{~kg} / \mathrm{m}^{3}$ ，直 径约 $40 \mathrm{~m}$, 在目标一连体坐标系下描述其相对质心转 动惯量为

$\mathbf{J}_{b}=\left[\begin{array}{ccc}401920 & -34851 & 42465 \\ -34851 & 381280 & 12460 \\ 42465 & 12460 & 438720\end{array}\right] \cdot 10^{3}\left(\mathrm{~kg} \mathrm{~m}^{2}\right)$.

目标初始旋转角速度:

$\boldsymbol{\omega}_{b 0}=\left[\begin{array}{lll}0.2 & 0.4 & 0.15\end{array}\right]^{\mathrm{T}}(\mathrm{o} / \mathrm{s})$.

目标惯量比矩阵在连体系下描述为

$\widehat{\mathbf{J}}_{b}=\left[\begin{array}{ccc}0.9161 & -0.0794 & 0.0968 \\ 0.0794 & 0.8691 & 0.0284 \\ 0.0968 & 0.0284 & 1\end{array}\right]$.

航天器主轴惯量比矩阵在连体系下描述为
$\widehat{\mathbf{J}}_{\Lambda}=\left[\begin{array}{lll}1.0659 & & \\ & 0.9415 & \\ & & 0.7778\end{array}\right]$.

按 $\mathrm{Z}$ 元素归一化的系统角动量比为

$\widehat{\mathbf{H}}_{a}=\left[\begin{array}{lll}0.9184 & 1.8593 & 1\end{array}\right]^{\mathrm{T}}$.

持续跟踪观测小天体连体系下三个特征点，构成 两矢量如下:

$$
\begin{aligned}
& \mathbf{r}_{b 1}=\left[\begin{array}{lll}
16 & -25 & 19
\end{array}\right]^{\mathrm{T}}(\mathrm{m}), \\
& \mathbf{r}_{b 2}=\left[\begin{array}{lll}
28 & 12 & -15
\end{array}\right]^{\mathrm{T}}(\mathrm{m}) .
\end{aligned}
$$

设视觉测量在百米处能够达到毫米级观测精度, 分别引入两项高斯噪声误差 $\varepsilon_{1}, \varepsilon_{2}$, 误差均值为 0 , 方 差为

$\operatorname{Var}\left(\varepsilon_{1}\right)=\left(0.1 \% \cdot\left|\mathbf{r}_{b 1}\right|\right)^{2}$,

$\operatorname{Var}\left(\varepsilon_{2}\right)=\left(0.1 \% \cdot\left|\mathbf{r}_{b 2}\right|\right)^{2}$.

辨识解算的考虑噪声的观测输入为

$\mathbf{r}_{a 1}=\mathbf{r}_{a 1}+\varepsilon_{1}$,

$\widehat{\mathbf{r}}_{a 2}=\mathbf{r}_{a 2}+\varepsilon_{2}$.

通过辨识的姿态转换矩阵将角速度换算到惯性 系，与动力学模型仿真计算得到的惯性系理想角速度 对比如图15, 辨识并滤波后的空间非合作目标角速度 在惯性系下轨迹如图16.

通过本文方法获得识别的角动量比与识别误差、
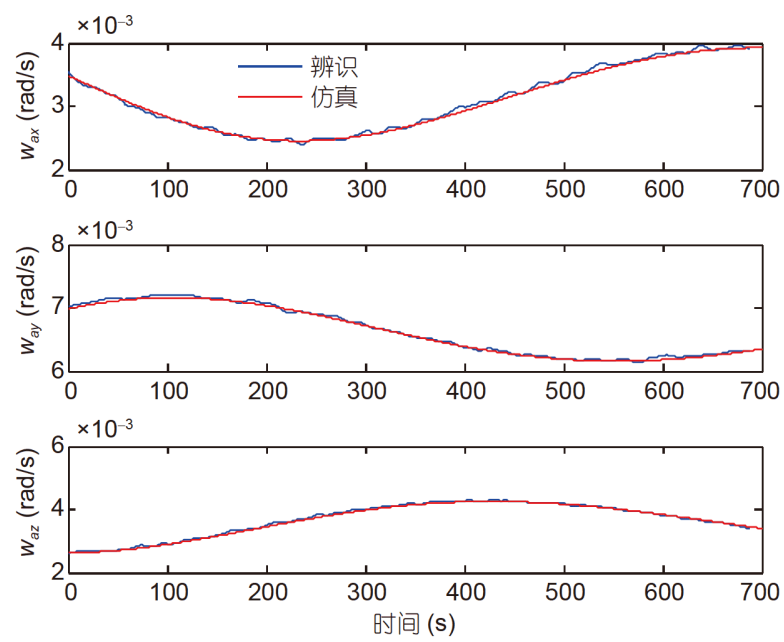

图 15 (网络版彩图)考虑噪声的惯性系下角速度对比

Figure 15 (Color online) Contrast of angular velocities with noise on the absolute coordinate system. 


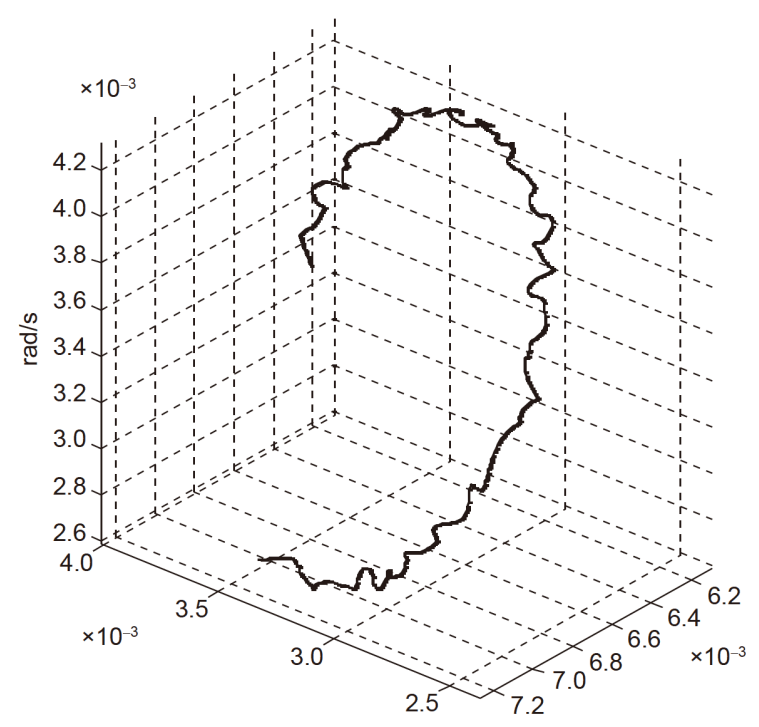

图 16 滤波后的惯性系下角速度轨迹

Figure 16 Trajectory of angular velocity after filter on the absolute coordinate system.

相对连体系惯量比与主轴系惯量比、相对连体系惯量 比, 主惯量比识别误差分别如下:

solve_$\widehat{\mathbf{H}}_{a}=\left[\begin{array}{lll}0.9126 & 1.8428 & 1\end{array}\right]^{\mathrm{T}}$, error_. $\widehat{\mathbf{H}}_{a}=\left[\begin{array}{lll}0.62 \% & 0.89 \% & 0\end{array}\right]^{\mathrm{T}}$, solve_ $\widehat{\mathbf{J}}_{b}=\left[\begin{array}{ccc}1.0122 & -0.0789 & 0.0843 \\ -0.0789 & 0.9437 & 0.0469 \\ 0.0843 & 0.0469 & 1\end{array}\right]$,

$$
\begin{aligned}
& \text { solve_ } \widehat{\mathbf{J}}_{A}=\left[\begin{array}{lll}
1.0968 & & \\
& 1.0164 & \\
& & 0.8427
\end{array}\right] \text {, }
\end{aligned}
$$

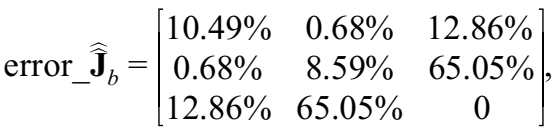

$$
\begin{aligned}
& \text { error_恿 }=\left[\begin{array}{lll}
2.9 \% & & \\
& 7.96 \% & \\
& & 8.35 \%
\end{array}\right] \text {. }
\end{aligned}
$$

\section{5 结论}

本文提出了一种仅基于立体视觉的解算空间非合 作目标相对姿态、角速度和惯量比的快速方法. 该方 法以双目视觉拍摄序列图像获取的特征点作为输入, 通过不变平面与能量球推导得到角动量与惯量比以及 主惯量比, 方法计算规模小, 适用于星上资源严重受限 条件下实现目标运动状态与惯性参数的快速识别. 目 标仅翻滚一圈以内即可获得参数辨识结果, 通过仿真 分析发现在考虑噪声的情况下算法依然是有效的. 目 标主轴惯量比的识别准确率能够达到约 $90 \%$ 以上，但 是对目标的本体系惯量比, 尤其是量级较小的惯性积 部分, 识别效果不好, 并且该方法非常重要的环节是 图像处理算法必须保证良好且精确的目标特征点信 息. 本文为小天体探测过程运动状态与参数辨识星上 快速实现提供了一种新的思路.

\section{参考文献}

1 Li J F. Special issue: Dynamics and control for asteroid exploration (in Chinese). Sci Sin-Phys Mech Astron, 2019, 49: 084501 [李俊峰. 小行星 探测动力学与控制专题·编者按. 中国科学: 物理学 力学 天文学, 2019, 49: 084501]

2 Wang L W, Ge S C, Jiang W S. Research on lander adhering and recovery technology for asteroid exploration (in Chinese). Spacecr Recov Remote Sens, 2019, 40: 14-23 [王立武, 戈嗣诚, 蒋万松. 小天体探测器着陆附着技术研究. 航天返回与遥感, 2019, 40: 14-23]

3 Wang S, Gu Z X. Development of small body exploration (in Chinese). Space Int, 2017, 7: 10-16 [王帅, 谷知行. 小天体探测发展态势. 国际太 空, 2017, 7: 10-16]

4 Gao L. Key technologies and characteristics of small body exploration (in Chinese). Space Int, 2017, 7: 17-22 [高否. 小天体探测关键技术和特 点. 国际太空, 2017, 7: 17-22]

5 Cui P Y, Jia H, Zhu S Y, et al. Research progress on optical navigation feature recognition and extraction technologies for small body exploration (in Chinese). J Astronaut, 2020, 41: 880-888 [崔平远, 贾贺, 朱圣英, 等. 小天体光学导航特征识别与提取研究进展. 宇航学报, 2020, 41: $880-888]$

6 Aghili F, Parsa K. Motion and parameter estimation of space objects using laser-vision data. J Guid Control Dyn, 2009, 32: 538-550

7 Shtark T, Gurfil P. Tracking a non-cooperative target using real-time stereovision-based control: An experimental study. Sensors, 2017, 17: 735

8 Hou X, Ma C, Wang Z, et al. Adaptive pose and inertial parameters estimation of free-floating tumbling space objects using dual vector 
quaternions. Adv Mech Eng, 2017, 9: 168781401771421

9 Ma C, Dai H, Yuan J. Estimation of inertial characteristics of tumbling spacecraft using constant state filter. Adv Space Res, 2017, 60: 513-530

10 Wang X, Wang Z, Zhang Y. Stereovision-based relative states and inertia parameter estimation of noncooperative spacecraft. Proc Inst Mech Eng Part G J Aerospace Eng, 2019, 233: 2489-2502

11 Ge D, Wang D, Zou Y, et al. Motion and inertial parameter estimation of non-cooperative target on orbit using stereo vision. Adv Space Res, 2020, 66: 1475-1484

12 Wang D Y, Zhang L, Zhu W H, et al. Autonomous relative navigation for noncooperative target using stereo vision measurements (in Chinese). Sci Sin-Phys Mech Astron, 2019, 49: 024509 [王大轶, 张否, 朱卫红, 等. 基于双目光学图像信息的空间非合作目标自主相对导航方法. 中国 科学: 物理学 力学 天文学, 2019, 49: 024509]

13 de la Fuente Marcos C, de la Fuente Marcos R. Asteroid (469219) $2016 \mathrm{HO}_{3}$, the smallest and closest Earth quasi-satellite. Mon Not R Astron Soc, 2016, 462: 3441-3456, arXiv: 1608.01518

14 Li X Y, Qiao D, Huang J C, et al. Dynamics and control of proximity operations for asteroid exploration mission (in Chinese). Sci Sin-Phys Mech Astron, 2019, 49: 084508 [李翔宇, 乔栋, 黄江川, 等. 小行星探测近轨操作的轨道动力学与控制. 中国科学: 物理学 力学 天文学, 2019, 49: $084508]$

\title{
Estimation of motion state for small celestial body detection based on sequence images
}

\author{
DENG RunRan, GE DongMing*, SHI JiXin, ZOU YuanJie, \\ ZHU WeiHong \& LI JiaNing
}

Beijing Institute of Spacecraft System Engineering, Beijing 100094, China

\begin{abstract}
Recently, with advances in aerospace technology, most countries have gradually shifted their attention to detecting small celestial bodies, which belong to the noncooperative space target task. In contrast to the cooperative space target, the noncooperative space target has typical characteristics, such as no prior knowledge, no cooperative sign, and no communication. However, necessary information on the noncooperative space target must be acquired before capturing. Obtaining the attitude, angular velocity, and inertia parameters of targets without contacting them is becoming a prominent technology for detecting small celestial bodies. This work proposes a new method for estimating the attitude, motion, and inertia properties of an unknown noncooperative space object. Simulations are presented to verify the method for targets with different properties and states. Moreover, the accuracy of the ratios of the principal inertia parameters can reach $90 \%$. In some cases, the most important step is that the computer vision algorithm must assure good and precise information about the feature points on the target. In summary, this paper presents a promising and valuable method for identifying the motion and parameters of noncooperative space targets before the landing of small celestial bodies. The proposed method is highly efficient and suitable for spacecraft applications.
\end{abstract}

detection of small celestial bodies, non-cooperative target, parameters identify, sequence images

PACS: $05.45 .-\mathrm{a}, 95.10 . \mathrm{Eg}, 95.40 .+\mathrm{s}, 42.30 .-\mathrm{d}$

doi: $10.1360 /$ SSPMA-2021-0002 